\title{
Acercamientos al concepto de metodología bibliotecológica
}

\author{
Valentino Morales Ló PeZ \\ Proyectos de Estudios Sociales, Tecnológicos \\ y Científicos, Instituto Politécnico Nacional \\ e-mail: nafta@ servidor.unam.mx
}

\begin{abstract}
RESUMEN
Este artículo ofrece, en primera instancia una visión global de la situación de los obstáculos que se han debido enfrentar en la construcción de la teoría bibliotecológica, además de nombrar aquellas personas que han trabajado los aspectos teóricos de esta disciplina, poniendo especial énfasis en los que han prestado atención a la metodología de la bibliotecología. Posteriormente se hace un recuento del estado que guarda el concepto de metodologíabibliotecológica y se ofrece una visión de las consecuencias de tener un concepto bien definido y consensado. Para culminar con la presentación de una propuesta inicial de concepto, fundamentada en la etimología de metodología de la ciencia y apoyada en algunas de las reflexiones de los metodólogos de la ciencia.
\end{abstract}

\section{APPROACHES TO THE CONCEPT OF METHODOLOGY \\ IN LIBRARY SCIENCE VAlentino MORALES-López}

\section{ABST RACT}

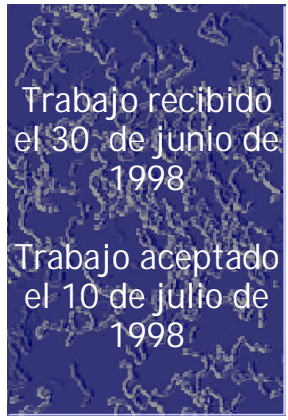

This articles offers, in the first place, a global vision of the obstacles that the construction of library science theory has had to face, as well as naming those people who have worked on the theoretical aspects of the discipline. Special emphasis is placed on those authors who have focussed their attention on library science methodology. Secondly, the article presents a state of the art review on the concept of library science methodology and offers a vision of the consequences of having a well-defined and consensual concept. The article ends with the presentation of an initial proposal of the concept, based on the etymology of scientific methodology and backed up by some reflections of the methodologists of science.

\section{INTRODUCCIÓN}

H n este trabajo se ofrece un esbozo general de la problemática que ha enfrentado la $\perp$ bibliotecología1 parala formación de un copusteórico, laubicación de su disciplina en la tipología de las ciencias y la formulación de la epistemología de la bibliotecología. Asimismo se hace mención de las personas, que de alguna manera han estado 
comprometidas en la conformación de una teoría bibliotecológica, poniendo énfasis en los autores de la segunda mitad del siglo XX, pues algunos de ellos hacen alusión al concepto de método o metodología, dentro de una temática más amplia y no sólo en referenciaa los procedimientos seguidos en la bibliotecología. No quiere decir que autores dásicoscomo Melvil D ewey no hayan trabajado siguiendo determinados métodos y teniendo presente cierto concepto de metodología, pero el tema de interés central es la conceptuación de la metodología bibliotecológica. Laproblemática enfrentada al ubicar los métodos del trabajo bibliotecológico se trata tomando en cuenta la discusión sostenida por los filósofos de la ciencia, especialmentelos del siglo XX, acerca de la metodología de la ciencia.

D e esta manera, se da un acercamiento general a las implicaciones en la teoría bibliotecológica, de existir un concepto de metodología bibliotecológica, bajo el supuesto de la inexistencia o insuficiencia del concepto usado, tomando en cuenta los acercamientos de los últimos años, por parte de los bibliotecólogos y reforzándolo con proposiciones provenientes de la filosofía de la ciencia. Se sitúa a la metodología bibliotecológica dentro de una posible e imaginaria epistemología bibliotecológica, que muestra que a pesar de su importancia acerca de retomar de otras disciplinas métodos y acercamientos conceptuales, es peligroso hacerlo sin una reflexión crítica, de tal manera que al momento de ser aplicados en el ámbito bibliotecológico sean transplantadas técnicas y métodos que no ayuden en la conformación coherente de los usados en la bibliotecología. Por esa razón al final se proponen dos conceptos basados en la etimología y descripción, que incluyan una reflexión sobre el concepto de metodología, con la finalidad de tener un punto de partida y avanzar en una discusión de mayor amplitud para visualizar otros aspectos enriquecedores del concepto de metodología bibliotecológica.

A simismo, otro interés es animar la discusión sobre el tema, al partir de la convicción de que los bibliotecólogos son gente preocupada por fortalecer a su disciplina de manera inteligente y con argumentos razonables, elementos que deben florecer en la discusión plural y tolerante.

\section{EPISTEMOLOGía Biblotecológica}

Por ser la bibliotecología una disciplina relativamente nueva, muchos de sus elementos constitutivos están en formación, por otro lado, históricamente el interés de los bibliotecólogos se ha centrado en la aportación de elementos para la solución práctica de asuntos que aquejan a la biblioteca. ${ }^{2}$ Aunado a lo anterior, sus labores se han remitido a la solución inmediata de problemas 0 a la adecuación del trabajo de la biblioteca ante nuevas situaciones enfrentadas por el desarrollo de la tecnología, ${ }^{3}$ en las áreas que afectan de manera directa el tratamiento y diseminación de la información. Ésas son algunas de las razones por las cuales se ha enfatizado la labor práctica, en detrimento de la reflexión y la conformación de un 
corpus teórico bibliotecológico, completo y maduro. Ello no implica la inexistencia de aportes teóricos, pero por lo general se abordan de manera periférica o se tocan asuntos muy particulares, sin aventurar propuestas con intenciones generales, o las propuestas son lanzadas desconociendo la epistemología y teoría del conocimiento, y llegan a ser una exposición de buenas ideas o lecturas, pero sin incorporarlas a un discurso propio y coherente. Tal aseveración no se puede aplicar a todos los autores, dado que existe un grupo de investigadores que ha trabajado en la conformación de la teoría bibliotecológica. Ejemplo de ello, en México, es el trabajo realizado en el Centro Universitario de Investigaciones Bibliotecológicas de la UNAM, con investigadores como Miguel Ángel Rendón, ${ }^{4}$ Ramiro Lafuente, ${ }^{5}$ Susana Sander, ${ }^{6}$ etcétera. En España están Emilia Currás ${ }^{7}$ y José López Yepes. ${ }^{8}$ Por parte de Cuba, existen investigadores como Emilio Setién ${ }^{9}$ y Salvador Gorbea. ${ }^{10}$ Brasil no se ha quedado atrás y sus investigadores han trabajado estos asuntos, como es el caso de Anna María Marques Cintra, ${ }^{11}$ Sebastiao de Souza12 y Solange Puntel Mostafa. ${ }^{13}$ Estados Unidos de América, con gran influencia en la bibliotecología mexicana, tiene en su haber una gran cantidad de autores, entre ellos se cuentan Charles H. Busha ${ }^{14}$ (con aportes a la metodología bibliotecológica), Jesse H. Shera ${ }^{15}$ (ya muerto, pero de importante referencia en la conceptuación de la bibliotecología durante la mitad del presente siglo), Herbert G oldhor, ${ }^{16}$ etcétera. Entre los representantes de Inglaterra está D . J. Foskett.17

Como parte de la bibliotecología inglesa se han contado a los autores hindúes, pero debido a la importancia de sus aportaciones se les nombra aparte, su figura representativa, sobre todo en la teoría de la clasificación, es S. R. Ranganathan.18

Es interesante observar que algunos autores, por ejemplo en el caso de México, caen en la categoría enunciada por K uhn, como at-sides ${ }^{19}$

\section{Problemática del Con CePto de metodología EN LA BIBLIOTECOLOGÍA}

Como se ha dicho con anterioridad, un área que no se ha construido con solidez y consenso entre los bibliotecólogos, es la epistemología bibliotecológica, pues se le ha prestado poca atención. En los acercamientos a formular tal epistemología, es de notar la pobre 0 nula reflexión sobre la mododoǵa bibidtedógica, pues la mayoría de los trabajos que toca el tema, se centra en la descripción de los métodos y técnicas a seguir o recomendables para la bibliotecología o al reflexionar sobre el tema, parten de una visión positivista. Entonces, algunos bibliotecólogos tienen la idea de la existencia del mótobdentífica ${ }^{20}$ vía única, sobre la cual, la validez de los productos de la investigación se apoya para darle a las diversas disciplinas status de ciencia (el método científico propuesto en bibliotecología pretende tener características del usado en las ciencias naturales, enraizando su tradición desde Galileo Galilei y Francis Bacon, aunque se basa en el propuesto por 
una corriente teórica de las ciencias sociales ${ }^{21}$ ). Pero las investigaciones sobre la metodología de la ciencia22 que han sustentado la posibilidad de la inexistencia del mátobdentíficuúnico, lleva a cuestionar el concepto de método bajo el cual se trabaja. Además, la investigación en bibliotecología se realiza mediante una gran diversidad de métodos ${ }^{23} 0$, en realidad cada quien hace la investigación como considera conveniente sin seguir método alguno, y sin saberlo se está implícitamente de acuerdo con la postura del anarquismo mtoddóġo propuesto por Paul Feyerabend. ${ }^{24}$ Sin embargo, la reflexión en torno a la metodología bibliotecológica se ha planteado en pocas ocasiones 25 en el ámbito bibliotecológico.

Lo cierto es que la metodología de la bibliotecología, la mayoría de las ocasiones, ha sido construida por personas con formaciones diferentes de la bibliotecológica, quienes aplican los métodos aprendidos en sus disciplinas de origen, ${ }^{26} 0$, los bibliotecólogos retoman métodos provenientes de otras disciplinas. En este sentido, un problema en torno a la metodología bibliotecológica es si los métodos usados por los bibliotecólogos han sido retomados de manera arbitraria de otras disciplinas ${ }^{27}$ y aun cuando sean aplicables a la bibliotecología, no se han comprendido a fondo y su aplicación se hace sin tomar en cuenta las circunstancias de la bibliotecología y, en ocasiones, el interés sólo está en reproducir un bellomítodo siendo una técnica lo que se estaría poniendo en operación, ${ }^{28}$ por eso, cabría aceptar la posibilidad de una asimilación de los métodos foránees ${ }^{29}$ como complemento y apoyo a los métodos ya existentes y usados por los bibliotecólogos en la conformación de su disciplina. E so, si se cree en la existencia de una metodología bibliotecológica, en donde metodología se entiende como el conjunto de métodos o procedimientos usados en una actividad o disciplina en particular.

La reflexión sobre la metodología bibliotecológica afecta la percepción acerca de la ubicación de la bibliotecología en la clasificación de las ciencias. ${ }^{30}$ Entre los asuntos que son de llamar la atención, para ser precavidos en la aseveración respecto a la ubicación de la bibliotecología dentro de la clasificación científica, se debe tomar en cuenta la interdisciplinariedad ${ }^{31}$ de la bibliotecología. Esta idea se fundamenta en la diversidad de asuntos tocados por la bibliotecología y que tienen relación con diferentes áreas del saber. Además al hacer un recuento de su historia, la bibliotecología en sus inicios está identificada con las disciplinas humanísticas (razón por la cual la Licenciatura en Bibliotecología se imparte en la Facultad de Filosofía y Letras de la UNAM). Pero a mitad del siglo XX, aproximadamente, se dio un fuerte impulso a ubicarla en las ciencias sociales y pueden observarse los métodos y conceptos provenientes de las ciencias sociales, aplicadas en la teoría y práctica de la bibliotecología y hay valiosas propuestas ${ }^{32}$ que ubican a la bibliotecología dentro de las ciencias sociales. Aunque a finales de la década de 1970, cobra fuerza la intención por llevarla a las denias duras En este sentido existe la preocupación de llegar a convertir a los bibliotecólogos en meros tecnólogos, dejando de lado la inspiración humanística de la disciplina, y la expresa D eanna B Marcum.33 Por eso es necesario señalar que muchas de las áreas de la 
bibliotecología, ${ }^{34}$ es difícil circunscribirlas únicamente a las ciencias sociales, ya que su inspiración viene de las humanidades ${ }^{35} 0$ de las llamadas dencas duras ${ }^{36}$ Ejemplo de ello y a decir de Shera, ${ }^{37}$ entre los orígenes de la bibliotecología en los Estados Unidos de América, está la original inspiración de las bibliotecas durante el siglo XIX, cuando el interés era promover la cultura para todos los miembros de la sociedad, al parecer relacionada con una concepción humanista; del otro lado, está la actual vinculación con la tecnología en la aplicación de elementos desarrollados por la computación, que han afectado la práctica y aun la concepción de las bibliotecas;38 además, otros pretenden ubicarla en las denias duras y se lanzan a la aventura de formular leyes de proposiciones bibliotecológicas, enunciadas mediante modelos expresados en términos matemáticos. ${ }^{39}$

Las observaciones anteriores no quieren decir que este trabajo, se incline por la relatividad en la bibliotecología, pero la problemática enfrentada en esta disciplina es la pauta para presentar una propuesta dentro de una discusión más amplia y por ello es necesario apuntar al estudio de temas sustanciales, pues su definición en estos momentos tendrá importantes repercusiones en el futuro. Siendo realistas no hay un consenso general para ubicar a la bibliotecología, además de que el término ha sido fuertemente impugnado y está sujeto a revisión, ofreciéndose alternativas como Cienaas dela Informadón ${ }^{40}$ A unque para la ubicación de la bibliotecología en la clasificación de las ciencias - ya sea en las ciencias sociales, las humanidades, 0, la tecnología-, es importante tomar en cuenta la propuesta expuesta por Jean François Lyotard, cuando habla de las tecno-ciencias ${ }^{41}$ y evaluar la posibilidad de ubicarla en esa propuesta.

Asimismo, este trabajo surge con la inquietud de que la metodología es uno de los elementos vitales en la conformación de una disciplina (aunque no necesariamente le dé carácter de ciencia) al darle rigurosidad y sistematización a su investigación y quehacer cotidiano. El conocer y comprender la metodología de la ciencia es de suma importancia para no encajonarla en una sola vía, sino ser abiertos a todas las posibilidades, analizándolas de manera crítica y retomando los elementos que puedan enriquecer a la bibliotecología. Esto, porque en varios de los trabajos ${ }^{42}$ sobre metodología bibliotecológica, revisados hasta el momento, da la impresión de que lo sustancial de la metodología estriba nada más en la aplicación de adecuados métodos de medición (vinculados especialmente con la estadística), dejando de lado elementos importantes ${ }^{43}$ para la metodología de la ciencia y que son útiles para la metodología bibliotecológica. Además, poco se toma en cuenta que a pesar de la utilidad de los métodos de medición, no son el único apoyo para realizar investigación.

Aquí se debe enunciar la existencia de una metodología propiamente bibliotecológica, la cual ha sido conformada desde los primeros indicios de un quehacer bibliotecológico, pero dado el desarrollo de la metodología científica positivista, se menospreció al grado de considerarla sin carácter científico. En apoyo a la idea de la existencia de métodos en la bibliotecología, se debe mencionar que la mayo- 
ría de las disciplinas primero han creado sus métodos y los han forjado de manera empírica y posteriormente se han dado a la tarea de reflexionar sobre esos métodos, aunque en algunas ocasiones la reflexión se da a la par, sobre todo con el interés de justificar la validez del uso de esos métodos.

\section{METOdOLOGÍA BIBLIOTECOLÓGICA}

Ante el estado del concepto demtodbogáa biblicteclóg்ca, mostrado con antelación, es necesario formular un concepto fundamentado en la etimología y descripción, además de tomar en cuenta las propuestas de los metodólogos de la ciencia. Por esa razón, el concepto presentado en este trabajo tiene esas características.

El término Meoddoǵa está compuesto por las siguientes palabras: 1) método, conformada por los vocablos griegos mtha - en medio, en seguida, a continuación, después- y odos-camino-;2) logía, del griego logos-tratado o estudio-. Por lo tanto, en su sentido etimológico se trata del estudio del método y/ o los métodos. 0 tra acepción usada es método y/ o conjunto de métodos de la actividad humana; el último significado posible es la aplicación de un método o métodos.44 Éstas son acepciones generales atribuidas al concepto de metodología.

Ahora bien, en la metodología de la ciencia, se han propuesto significados específicos o diferentes y son: a) la lógica o parte de la lógica que estudia los métodos; b) el conjunto de métodos empleados por una ciencia o grupo de ciencias; c) el estudio filosófico de los métodos de una(s) ciencia(s) o sólo del método. ${ }^{45} \mathrm{La}$ primera acepción no se comentará en este artículo, porque el interés primordial es el aspecto conceptual, no el instrumental, preocupación básica para la lógica. Entonces brevemente se comenta sobre las otras dos acepciones, que afectan de manera directa la noción del concepto de metodología.

La acepción a tratar será la tercera, pues lleva a una discusión que para algunos resulta estéril, pero es justificable porque en la literatura bibliotecológica consultada, se menciona el método científico como si su existencia no fuera motivo de discusión. Es importante comentar cuáles son las posiciones sostenidas en la filosofía de la ciencia. Cierto es que la ontología del método científico desde su establecimiento como regulador de la investigación científica, ${ }^{46}$ no fue problema sino hasta el siglo XX.

Son varias las posturas en torno al método científico, una es la sostenida por Paul Feyerabend, quien conmocionó a la filosofía de la ciencia, al enunciar la inexistencia del método científico, proponiendo para el trabajo en la ciencia el todbse vale $^{47} \mathrm{O}$ tra posición importante en este siglo y con una mayor tradición, es la sostenida por el Círculo de Viena, identificada como neepositivismo que considera que los frutos de la ciencia, tangibles aún en la vida cotidiana del ser humano, indican 
su existencia, pues sólo mediante el apoyo del método científico general, único y con unidad, se lograron todos los triunfos de la ciencia. Además está la posición de Karl Popper ${ }^{48}$ e Imre Lakato ${ }^{49}$ quienes creen en la existencia del método científico, mas no en su carácter único. ${ }^{50}$ Popper propone la falsación como instrumento metodológico para valorar el carácter científico de las teorías. Lakatos considera que el desarrollo metodológico de la ciencia se ha dado mediante programas de investigación científica.

En este trabajo, se toma una postura no extremista, considerando que el método científico no existe per sey no puede ser exhaustivamente especificado en un sistema articulado por reglas. Su existencia depende del proceso seguido por los científicos en su trabajo cotidiano, pues usan ciertos procedimientos bajo los cuales regulan su trabajo (no quiere decir que el seguimiento estricto de esos procedimientos hará válidos los resultados de la investigación o les da categoría de conocimiento científico). Entonces, se habla de un método científico de acuerdo con el contexto en el cual está ubicado el investigador. Pues el lenguaje único y científico, además de las supuestas reglas del método científico, es convencional y transformable. ${ }^{1}$ Por lo tanto, el método científico es el conjunto de procedimientos seguidos por el(los) científico(s) en la investigación para justificar la verdad de teorías y/ o modelos que intentan representar la realidad, pero no es universal, siendo diferente en cada área del saber.

Respecto a la segunda acepción y ya fijada la posición conforme a la ontología del método científico, puede decirse que los métodos de la ciencia no es posible restringirlos a un conjunto exhaustivo de determinados métodos; aunque existen varios métodos identificados como útiles para la ciencia: deductivo; inductivo; analítico y sintético; observación; sistemas formales, modelos y representación de los hechos; la medida; hipótesis; probabilidad; axiomático; de concordancia; hipotético-deductivo; experimentación; comprobación o corrección; y el de prueba y error. ${ }^{2}$ Por lo tanto existe una gran diversidad de métodos en la ciencia y el científico echa mano de ellos, de acuerdo con el contexto en el que está ubicado y el tipo de investigación en la cual está involucrado.

De lo anterior se desprende que el concepto de metodología bibliotecológica puede referirse al estudio del método y/ o los métodos usados en la bibliotecología; al método y/ o conjunto de métodos de la bibliotecología; la aplicación de un método o métodos en la bibliotecología. En un segundo nivel, el método científico en la bibliotecología se debe implementar de acuerdo con el contexto de esta disciplina, pues se trata del conjunto de procedimientos seguidos por los bibliotecólogos, siendo diferente en cada área de esta disciplina. Por ejemplo: en la bibliometría se usa la estadística, la clasificación echa mano de la teoría de conjuntos y la lógica, en los encabezamientos de materia se usa la hermenéutica, etcétera. Además los métodos usados en la bibliotecología no pueden restringirse a un conjunto exhaustivo de determinados métodos, aunque es posible identificar los 


\section{InveticaaónBiblictedógica v. 12 No. 25 julio/ diciembre de 1998}

de uso más común y las áreas donde son aplicados, para sugerir una tipología que pueda regir la investigación bibliotecológica.

Como se ha visto hasta este momento, no es factible tomar una postura reduccionista respecto a la metodología bibliotecológica, dado que han existido posiciones diversas. Son varias las líneas que se deben trabajar para arribar a concepciones claras sobre la metodología bibliotecológica. Por un lado es necesario remitirse al estudio de la metodología de la ciencia y de manera crítica explorar cuáles elementos conceptuales de importancia puede aportar en la conformación del concepto manejado en bibliotecología; se remarca que de manera crítica, ya que se tiene conocimiento del esfuerzo realizado por retomar métodos de otras disciplinas para la bibliotecología, sin embargo, no se consideraba que la bibliotecología tuviera características particulares que la hicieran diferente de las otras disciplinas y por ello la aplicación de esos métodos, se realizaba a nivel técnico. También es necesario revisar el trabajo que se ha desarrollado en la literatura bibliotecológica, pues no es posible menoscabar el esfuerzo de otras personas, cuyas aportaciones lograron hacer llegar a la bibliotecología hasta el estado actual. Por último de manera concertada plantear esta problemática en los diferentes foros donde se tenga oportunidad, ya que cada bibliotecólogo es capaz de ofrecer una opinión valiosa, por su experiencia profesional o en su trabajo como investigador de la bibliotecología.

\section{CONCLUSIONES}

A lo largo del trabajo se ha observado la variedad de implicaciones de la metodología bibliotecológica. O bviamente, el concepto de metodología bibliotecológica presentado en este trabajo, apenas abre la puerta para una discusión más amplia, la cual debe darse en los diferentes ámbitos de la disciplina.

La discusión debe abarcar los siguientes aspectos:

* Estudiarafondo laliteratura bibliotecológica sobremetodologíapara reconocer qué es lo que se tiene bien fundamentado y de esa manera reconocer la tra dición que antecede a esta época. También será forzoso revalorar el carácter dentífico de la bibliotecología.

* Clarificar adecuadamente el concepto de metodología bibliotecológica, apoyado en las propuestas de la metodología de la ciencia.

* Proponer una tipología para clasificar los métodos usados en cada área de la bibliotecología, pues así se conocerán y mejorarán.

- Analizar de manera exhaustiva los métodos retomados de otras áreas del sa ber para reconocer su grado de aprovechamiento para el trabajo bibliotecológico. 
1. El término bibliotecología está en discusión, pues en la actualidad se han propuesto otros términos como el de ciencia de la información. La razón para usarbiblideedoǵaen este trabajo, es por el convencimiento de que es el concepto con el que hasta el momento la sociedad identifica a los profesionales que trabajan conbibidtecas, docmentos, informaiónyconocimientosquebuscanlossereshumanosenesosdboumentosydeadividadesdelaskiblide cas para que esosseres humanos dotengan esa infomaaóny conocimientoquerequieen, aæandodlas mismascietosconoimientosydrosdbumentos(Miguel Ângel Rendón Rojas. Basestéricasyfilosóicas delabiblictedoǵa 132 p. p. 36). Ante esto se opone el argumento de que la bibliotecología se ha modificado durante su trayectoria histórica y ese concepto ya no es suficiente para abarcar todas las actividades identificadas con esa disciplina, pero la disciplina no necesariamente cambia, sino más bien sus procesos 0 aproximaciones al objeto de estudio. Aunque se objetará que al transformarse las estructuras cognitivas para el estudio de determinado objeto o fenómeno, a pesar de que el objeto continúa siendo el mismo, es necesario encontrar o identificar un nuevo concepto. Pero la esencia (acercándose demasiado a Giordano Bruno) de las cosas que se estudian continúa siendo la misma y lo único que se modifica es el discurso característico en la bibliotecología, puesto que a pesar de emplear términos rimbombantes, en la práctica cotidiana y la enseñanza, los bibliotecólogos continúan trabajando con los mismos asuntos, y si bien tecnológicamente han existido modificaciones asombrosas, el objeto de estudio y los procesos no han sufrido una modificación sustancial.

2. Esta situación se puede constatar con algunas de las tesis producidas por los egresados del Colegio de Bibliotecología de la Facultad de Filosofía y Letras de la UNAM, la Escuela Nacional de Biblioteconomía, en México, y aun de varios de los productos de las investigaciones realizadas en el Centro Universitario de Investigaciones Bibliotecológicas.

3. El caso que puede mencionarse es el impacto del vertiginoso desarrollo de las computadoras en la práctica bibliotecológica. Ante esto se ha producido una vasta literatura, desde mediados de la década de 1980 hasta este momento. Con ello han aparecido nuevos términos que son meros anglicismos, trasladados, de manera arbitraria al lenguaje español y a pesar de reflejar un cambio en ciertos procedimientos de la bibliotecología, no necesariamente aportan en el terreno conceptual o hacen una diferencia en la esencia de un proceso que se realiza con o sin apoyo de la tecnología.

4. Miguel Ángel Rendón Rojas. "Algunas peculiaridades de la ciencia bibliotecológica".

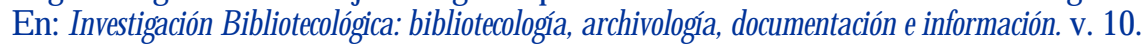
no. 21 (1996): 22-26. Bases ténicasyfilosóficas delabiblicteedoǵa 132 p. "Hacia un nuevo paradigma en bibliotecología". En: Transinformagaa v. 8. no. 3 (1996): 17-31. "La información como ente ideal objetivizado". En: Invetigadón Biblicteedógica: biblidteedoóa, ardivdoǵa, documentacóneinfomaaón v. 9. no. 18 (1995): 17-24. "Metodología de la investigación en bibliotecología". En: Investigaaón Biblictedoógica : biblidtecoloǵa, ardivdoóa, dbaumetacóneinfomación v. 10. no. 21 (1996): 27-29. "Las tareas para la fundamentación de la bibliotecología”. En: InvestigaiónBiblideedógica: biblictedogáa, ardivdoǵa, dbar metacoón einformacón v. 10, no. 20 (1996): 9-15.

5. Ramiro Lafuente. "¿Es necesario un nuevo paradigma en catalogación?” En: Invetigat

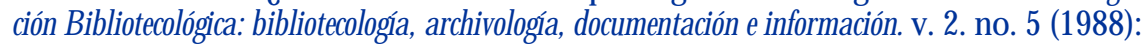
4-11. "La síntesis crítica del conocimiento bibliotecológico: su valor para la investiga- 
ción en Bibliotecología". En: Investigaaón Biblideedógica: biblidtedogóa, archivologá, dbar mentacóneinformacón v. 2, no. 4 (1988): 3-10. En coautoría con estela Morales Campos. "Reflexiones en torno a la enseñanza de la bibliotecología". En: InvestigaiónBibidteedó gia: biblictedogóa, ardivoloǵa, dbammtadón einfomacón v. 6. no. 12 (1992): 25-33.

6. Susana Sander. "Clasificación ¿actividad técnica o teórica?" En: InvestigaaónBiblidtedó gica: biblidtedogóa, archivoloǵa, doumetacióneinformacón v. 2. no. 5 (1988): 43-50. "Elementos histórico-teóricos para la indagación de la estructura teórica de la Biliotecolo-

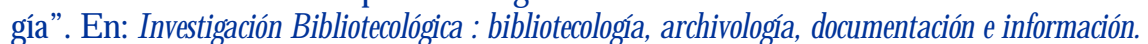
v. 3. no. 8. (1990): 31-37. "El problema de lainvestigación bibliotecológica norteamericana : una revisión (1930-1960)”. En: InvesticadónBiblicteedógica: biblicteedoǵa, ardivdo gá, doumentacoón einfomaaión v. 8, no. 16 (1994): 20-24.

7. Emilia Currás. Lasdieniasdeladbamentadón: biblidtedooga, ardivdogá, dbamentadóneinformacón Lainfomacón en susnuevos aspetos 307 p.

8. José López Yepes. "El concepto de ciencia de la documentación: unidad en la diversidad o diversidad en la unidad". En: InvesigadónBiblicteedógica: biblidteedoǵa, archivoloǵa, dbamentaaóneinfomaaón v. 10. no. 21 (1996): 4-6. Ladoumentacóncomodisaiplina: teeńae histona. 2a ed. 337 p. El estudiodeladbaumentación: mudodooǵaybibliografíafundamental. 185 p.

9. Emilio Setién y Salvador G orbea Portal. "D e la Bibliotecología al sistema de conocimientos científicos bibliológico-informativo". En: InvestigaáónBiblicteedógica: biblicteo logáa, archivdoǵa, dbamentacoóneinfomadón v. 8. no. 16 (1994): 21-25. "Estudio de desarrollo de las bibliotecas públicas cubanas: condiciones para la modelación matemática

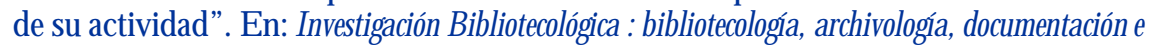
informadón v. 5. no. 11 (1991): 24-32. "El objeto de estudio de las disciplinas bibliológico-informativas y su enfoque en la Biblioteca Nacional José Martí de Cuba". En:Investigacoón Biblictedoógica: biblidtedogáa, ardivdogáa, dbametaaón einformacón v. 10. no. 21 (1996): 7-13.

10. Salvador G orbea Portal. "Principios teóricos y metodológicos de los estudios métricos de la información". En: Investigaáón Biblideedógica: biblicteedogáa, archivdoǵa, dbar mettacóneinfomacoón v. 8. no. 17 (1994): 23-32.

11. Anna María Marques Cintra. "Subjetividade e interdisciplinaridade na Biblioteconomia”. En: Transinfomaçaa v. 8. no. 3 (1996): 32-43.

12. Sebastiao de Souza. Dimensioes atuaisdabilideeconma mo Brasil: umestudbatravés desuas tendenaas 160 p. "Fundamentos filosoficos da biblioteconomia". En: Biblidteen v. 14. no. 2 (1986): 189-196. "Información: utopía y realidad de la Bibliotecología". En:

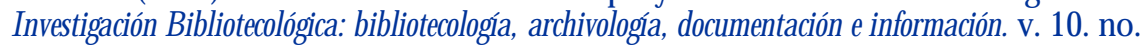
21 (1996): 14-17.

13. Solange Puntel Mostafa. "Enfoques paradigmaticos da bibliotecologia: unidade na diversidade ou diversidade na unidad. En: Inveticgaión Biblictedógica: biblideedoóa, ardivdogá, dbametaaión einfomacón v. 10. no. 21 (1996): 18-21. "Filosofando sobre a área de informaçao". En: Londina, SimpósioBrasil-sul deinfomaçao 12 p. (copia xerox) maio, 1996. 
14. Charles H. Busha. Resench ntools in libranianship techniques and integretation $417 \mathrm{p}$. (Versión en español: Médodos deinvestigadón en biblidtedoǵa : ténicas einteppezadón 408 p.)

15. Jesse H. Shera. Losfundamentos dela eduraaónbibidteclógica Introdurtion tolibraryscience basicdements oflibrarysience

16. Herbert G oldhor. Anintroductiontosaientificrearch in libranianship

17. D . J. Foskett. “The classification research group, 1952-1962". En: Libri. v. 12, no. 2 (1962): 127-138. "The communication chain". En: Theinfomation environment: a wodd view

18. Shiyali Ramamrita Ranganathan. Thefivelaus of librarysaience 1989. 449 p.

19. Los at-sidesson individuos que provocan cambios en o del paradigma de una disciplina (Wordd dhanges: Thomas Kuln and thenature of science vi, $356 \mathrm{p}$. Se trata de personas ajenas al paradigma dominante, ya sea por una formación diferente, propuestas novedosas, visiones críticas, personalidad creativa, identificación precoz, sin prejuicios, imaginativo, con pensamiento divergente, libre para partir en direcciones diferentes, rechaza la solución antigua y toma una dirección nueva, etc.; y en algunos casos se trata de gente joven. Tomado de: Thomas S. Kuhn. La tensión esenaal: etudios sdeetos sdrela tradiaónye cambioen ém ámbtodela denaa / tr. de Roberto Helier. p. 232, 248249.

20. Charles H. Busha. Ibidam p. 5. G oldhor, Herbert. Ibidem p. 9. A librarysiencerearch reader and bibliographic guide ed. Charles H. Busha. Colorado : Libraries Unlimited, 1981. 201 p. p. 1. O ctavio G. Rojas L. "La investigación y el desarrollo integral de la bibliotecología en la región". En: Memria de SeninariopreConferencia IFLA sdreetura dón paralainvestigaióneinnestigaión para la eeduradón p. 59.

21. En especial se sigue la escuela de Estados Unidos, apoyada en la creencia de que toda investigación debe ser comprobable y siguiendo la utopía de la perfección de los números, proponen varios métodos estadísticos (aunque por el seguimiento que se les da, parecen técnicas), mediante los cuales, a su juicio, es posible comprobar la validez de las investigaciones, y (de manera prácticamente mágica) se refuerza la cientificidad de la disciplina en la que se trabaja. No por ello se debe proponer el rechazo de los métodos estadísticos, pero se debe ponderar su uso, de acuendo a lo que se esté trabajando. A library siencerench reader and bibliographic guided Ibidkm p. 21-22. Blaise Cronin. "Approches to market research". En: Infomation rearch : rearch mthoodsin libraryandinformation sience 123-138. p. 130. Charles H. Busha, Ilidam p. 5. E. W. Diarmid. Thelibrarysurvey: prodlems andmathods p.p. 1, 3. Herbert G oldhor, Ibidem p. 3-6, 14-32. Samuel D. Neill. Dilemmas in the study of information : exploing the boundanies ofinformation sience p. 140-155. Zaheta Bansic. "Availability and use of international organizations documentation in Yugoslav libraries". En: Ibidam: 82-104. p. 82.

22. Ana Rosa Pérez Ransanz. "Modelos de cambio científico". La denaa: estructurayde samdla pp. 181-202. Gérard Fourez. La construción de conoimientodentífica filosofía yética dela dena. p. 70, 110, 116. Newton-Smith. La racionalidaddela dena. p. 141, 153.

23. Charles D . Emery. Buyes and bonowes theapdication of consumer theery to thestudy oflibraryuse p. 1. 
24. Con esta aseveración es necesario tener cuidado, pues si bien Feyerabend en Contra d mítodby Tratadb contra e mítodo enarbola el tobsevale, posteriormente en La denaa en una sociedad libre pondera tal declaración, aseverando que no intenta sustitir un conjuntoderegas generalespar droconjunto, sino convencer al lector de que todas las metodologías, incluidas las más obvias, tienen sus límites, señalamiento hecho con anterioridad, pero que a su juicio, no había sido observado por sus críticos, atribuyéndosele la intención de sustituir unas reglas generales por otras. Paul Feyerabend. Contra e mítodb Ladienia en una sociedad libre p. 31-32. Tratadocontra d nútoob: es quema deuma teeńa anarquista de conoimietta p. p. 12, 17n, 174, 185, 206, 290.

25. Un caso, pero con un enfoque kuhniano, es el artículo ya referido de Susana Sander "El problema de la investigación en la bibliotecología norteamericana Op at.

26. Como Miguel Ángel Rendón, que propone el método dialéctico, con el cual es posible hacer uso de diferentes vertientes metodológicas, sin caer en un eclecticismo desafortunado o en extremos que impidan el buen desarrollo de la investigación. Retomado de una conversación con el autor y de Miguel Ángel Rendón Rojas. "Metodología..." Ilidam 27-29.

27. H. Curtis Wright. "Inquiry is science and librarianship". Jaumal of LibraryHistary. no. 13 (1978): 250-264.

28. Al estarse aplicando de manera algorítmica, sin posibilidades de modificación.

29. Hay varias propuestas en ese sentido y se puede ejemplificar con: Constance Ann Mellon. Naturalisticinquiry for librarysience: mthods and application for reserth eveluation and teaching I. K. Ravichandra Rao. Quantitativemthods for libraryandinformation sience 1983. $271 \mathrm{p}$.

30. Aceptando la posibilidad de poder clasificar a las ciencias, cuestión muy debatida, aunque existen algunos consensos mínimos primordialmente ubicados en un plano descriptivo.

31.Hay varias propuestas como interdisciplinaria, multidisciplinaria y transdisciplinaria. Se escoge interdisciplinariedad, en este caso, porque da la idea de la convergencia en una disciplina de elementos provenientes de diversas disciplinas.

32. Ejemplo de ello son: Miguel Ángel Rendón Rojas. Basesteénicasyfilosóicasdela Biblio teedoǵa Ilidem p. 36. O. S. Chubarian. Biblicteedoǵa general. p. 9-20, 42-44. Ronald R. Powell. Basicrearchmthods for libraians p. 1.

33.D eanna B Marcum. "Librarians or technicians?: which shall we be?". Informationfora newage reekfiningthelibraian pp. 11-14.

34. Como la catalogación, clasificación, bibliografía, indización, administración de bibliotecas, la tecnología aplicada a la consulta, los procesos técnicos del libro, etcétera.

35. Jesse H. Shera. Introduction tolibrarysaence basicdement of library saene Ibidam p. 9. Joseph P. Natoli. "Librarianship as a human science: theory, method and application". LibraryReserch no. 4 (1982): 163-174. p. 163-166.

36. Salvador G orbea Portal. El moddo matenático deBradford su aplicacón a las revistas latinoameicanas delas dienias biblidteedógicas ydela informadón

37. Jesse H. Shera. Introduction tolibrary saiene.. qp at. p. 33-40. 
38. Esta situación se revela en el lenguaje de boga en la actualidad. Pues se puede observar el uso de anglicismos y términos inexistentes, apenas una década atrás. Sin embargo se debe tener cuidado y no dejarse engañar con el canto de las sirenas, pues no por usar un lenguaje nuevo y atractivo, la bibliotecología necesariamente ocupa un nuevo statusy un lugar privilegiado dentro las disciplinas científicas. Ya que, como es bien sabido, el lenguaje no cubre todos los requerimientos que le dan a una disciplina el carácter de ciencia. Además, es bueno recordar la ironización de Nietzche, sobre la fragilidad de los conceptos humanos, los cuales son fáciles de crear y desechar.

39. C. E. Shannon y W. Weaver. Thenathematical theery of communication Emilio Setién. "Estado de desarrollo de bibliotecas públicas cubanas... Ibidem Rosa Ma. Martínez Rider y Agustín Gutiérrez Chiñas. "Reflexiones sobre los aspectos científicos de la investigación en Biblioteconomía". En: Investigadoón Biblidtedoóġa: biblidteedoǵa, archivdoǵa, dbamentacóneinformaión v. 2. no. 3 (1987): 60-62, p. 61.

40. Esto tiene relación con la disputa sobre cual paradigma debe prevalecer y se considera que los posibles paradigmas son el de serviobiblictearioo el de informaión como lo muestra el texto de Richard A postle y Boris Raymond Librarianshipand theinfomation paradign $162 \mathrm{p}$.

41. Jean Francois Lyotard. La postmodemidad explicada a los niños/ tr. Enrique Lynch. p. 29-32.

42. I. K. Ravichandra Rao. Quantitative mothoos for library and information sience Ibidem Quantitativem thoosinlibrarianship standards, rearch managment.

43. Como ejemplo, el análisis efectivo de los datos obtenidos en la investigación, pues la investigación científica no sólo se trata de la descripción de un fenómeno y la manera como llega a realizarse el trabajo estadístico en bibliotecología, sólo se remite al aspecto descriptivo.

44. Esos significados son a nivel general y pueden encontrarse en: Eli de G ortari. El mé tob delas dienias: nociones fundamentales p. 17, 89. Henry Van Laer. Phylosphy of saience p. 55. Mario Bunge. Lainvestigaióndentífica: su estrategjaysufilosofía p. 24.

45. Leoncio O rtiz González. Dicionario delógica p. 249. Mario Bunge. La investigaaón dentífica Ibidam p. 27, 50. Metoddoǵa dd conoimiento centífica 5a ed. 445 p. p. 4-5, 8-9. Sebastián Álvarez. Racionalidady mútodbcientífico Racionalidad quistérica

46. Haciendo un breve repaso en la historia de la filosofía de la ciencia, se encuentra que no es posible hablar estrictamente entre los griegos de un método científico, tal y como es concebido en la actualidad, pues ellos no tenían esa concepción, pero sí es posible ubicar el desarrollo de algunos procedimientos, identificados actualmente como métodos usados por la ciencia, tales como la mayéutica desarrollada por Sócrates y la lógica trabajada por Aristóteles. En la Edad Media, siguiendo con la tradición heredada de la cultura grecolatina, no existieron mayores aportaciones, salvo el reforzamiento de la lógica. Algunos señalan el surgimiento del método científico con el trabajo de $\mathrm{G}$ alileo y su pretensión de matematizar sus resultados, pero a quien se le reconoce como el que fundamenta la idea del método científico es a Francis Bacon. Otros filósofos y científicos con un trabajo sólido al respecto son D escartes, Newton, Mill, etc. Mayor información sobre este punto se puede obtener en: David Oldrey. El arcodi conoimienta introducción a la histona dela filosofía y mudodoǵa dela dena. 607 p. G. E. R. Lloyd. Methods andprdblensin Greek sience: sdeetedpapes 457 p. Javier 


\section{InveticaaónBiblictedógica v. 12 No. 25 julio/ diciembre de 1998}

Echeverría. Introduccóna la mtodbloǵa dela ȧena: filosofía dela ȧena end sigoXX. 322 p. Metodoloǵa dd conocimientodentífica Ibidam Ralph M. Blake, Curt J. D ucasse and Edward H. Madden. Theories of sientific mothod the Renaissancethrough thenineteenth century $346 \mathrm{p}$.

47. Véase la nota 24.

48. Uno de los filósofos con mayor reconocimiento en este siglo. Su obra de mayor interés para la metodología, se trata de La lớca delainvetigadón científica

49. Discípulo de Popper, muerto a temprana edad, sus trabajos fueron compilados en un libro denominado Los progamas deinvestigaióndientífica

50. Esta aseveración debe ser tomada con cuidado, sobre todo si se toma en cuenta que Popper propone a la falseación como metodología única para validar científicamente una teoría. Ante esto, sus defensores consideran que él propone, más no impone unilateralmente esa metodología, por lo que no se le puede acusar de concebir un método único.

51. Gérard Fourez. La construcción di conoimientodentífica Ilidam p. 26, 110, 116. W. H. Newton-Smith. La racionalidaddela denia. Ibidam p. 141, 153, 229-230, 250, 290-292. Sebastián Álvarez. Ibidkm p. 166.

52. - Eduardo Primo Yúfera. Introducción a la investigacón dentífica y teendógica p. 22-29. G érard Fourez. La construción dd conoimiento dentífico Ilidem p. 26-29, 32. Marx W. Watofky. Introdurciónalafilosofía dela denaia p. 153, 261. Metoddoǵa dd conoimientodientíica p. 6-7, 233-234, 240-241, 243-244, 246-250, 253, 273. Roberto Torrett. "El método axiomático". La denaia: structura ydesamollo 89-110. p. 89, 107. W. H. NewtonSmith. La radionalidaddela denaia Ilidem p. 24, 31.33, 242.

\section{BIBLIOGRAFÍA}

A librarysaenrerearchreader and bibliographicguide ed. Charles $\mathrm{H}$. Busha. Colorado : Libraries Unlimited, 1981. 201 p.

Álvarez, Sebastián. "Racionalidad y método científico". Racionalidadeqistérica/ ed. León O livé. Barcelona: Trotta, 1995. 278 p. (Enciclopedia Iberoamericana de Filosofía ; 9): 147-170.

Apostle, Richard y Boris Raymond. Libraianship and theinformationparadigm London : The Scarecrow Press, 1997. xiii, 162 p.

Bansic, Zaheta. "Availability and use of international organizations documentation in Yugoslav libraries". Infomation reserch : rearchmothodsinlibraryandinfomationsience/ ed. by $\mathrm{Nev} \mathrm{Tu}$ dor-Silovic and Ivan Mihel. - London : Taylor Graham, c1988. 261 p. - ISBN 0947568 26 3: 82-104.

Blake, Ralph M., Curt J. Ducasse and Edward H. Madden. Theo niesofscientificmthod: theRenaissancethraughtheninteenth century/ 
ed. By Edward H. Madden . - New Y ork : G ordon and Breach, 1989. viii, 346 p. - (Classics in the history and philosophy of science ; 2). - ISBN 288124-351-7 (France).

Bunge, Mario. Lainvestigaaóndentífica: suestrategiaysufilosfía / tr. Manuel Sacristas. 2a ed. Barcelona : Ariel, c1983. (Methodos : filosofía, historia, sociología y política de la ciencia y de la Técnica).

Busha, Charles H. Reserch motods in libranianship techniques and intenptation. New York : Academic Press, 1980. 417 p.

Chubarian, O. S. Biblictedoǵa geneal. - Cuba : Editorial Científico-Técnica, 1976. $367 \mathrm{p}$.

Cronin, Blaise. "Approches to market research". Infomation reardh rench mothoosinlibraryandinfomationsience/ ed. by Nev Tudor-Silovic and Ivan Mihel. - London : Taylor G raham, c1988. 261 p. - ISBN 094756826 3: 123-138. p. 130.

Diarmid, E. W. Thelibrarysuney. prodensandmothook - Chicago : ALA, 1940. $\mathrm{xv}, 243 \mathrm{p}$.

Echeverría, Javier. Introduciónala młoddoǵa dela ȧena: filosofía dela denia en d sigoXX. - Barcelona : Barcanova, 1989. 322 p. - (Barcanova: temas universitarios). - ISBN 84-7533-472-5.

Emery, Charles D. Buyes and bonowes theapdication of consumer thery to the study oflibaryuse New Y ork : The Haworth Press, 1982. 188 p.

Feyerabend, Paul. Contra d mítodb - Madrid : A riel, 1974.

- - . La äenaaenumasoceedadlibre/ tr. de Alberto Elena. - México : Siglo XXI, 1982. 261 p. - (Teoría).

- - Tratadbcontrad mítoda esquemadeuma teeńaanarquista da conoimienta México : REI, 1997. $319 \mathrm{p}$.

Fourez, Gérard. La construciónde conocimiento cietífica filosfía yética dela àerda Madrid : Marce, 1989. 206 p.

Goldhor, Herbert. An introduction to sientific rearch in libraniandip Illinois : University Press, 1972. 203 p.

G orbea Portal, Salvador. El moddomatemáticodeBradford su aplicaaón a las re vistas latincameicanas delas denias biblideedógicas ydelainfomadón México : UNAM, CUIB, 1996. $152 \mathrm{p}$.

G ortari, Eli de. El mítodbdelas äenias noiones fundamentales México : Grijalbo, imp1996. 151 p. (Tratados y manuales Grijalbo).

Kuhn, Thomas S. Latensiónesenal: etudiossdetossobrelatradioónye canbioen al ámbitodela denaia/ tr. de Roberto Helier. México : CONACYT : FCE, 1987. - 380 p. - (Sección de obras de ciencia y tecnología).

Laer, Henry Van. Phylosqphyofsience 2a ed. Pittsburgh : D uquesne University Press, 1963. 2 t. (Duquesne studies : philosophical series ; 6). 
Lloyd, G. E. R. Methodsandproblensin Grek saence sdeted papers Cambridge : Cambridge University Press, 1991. xiv, 457 p. ISBN 0-521-37419-7.

Lyotard, Jean Francois. La postmodemidadexplicada a los niños/ tr. Enrique Lynch. - Madrid: G edisa, 1991. 123 p.

Marcum, D eanna B. "Librarians or technicians? : which shall we be?" Infomationforanewage redfiningthelibranan Colorado : Libraries Unlimited, 1995. 192 p. pp. 11-14.

Martínez Rider, Rosa Ma. y Agustín Gutiérrez Chiñas. "Reflexiones sobre los aspectos científicos de la investigación en Biblioteconomía". InvestigadónBiblidtedóǵca: biblideedoǵa, archivdoǵa, doumentacón einfomadón v. 2. no. 3 (1987): 60-62.

Mellon, Constance Ann. Naturalistic inquiry for library saience mothoos and application for rearch, evaluation and teading $\mathrm{New}$ York: GP, 1990. 201 p.

Metoddoǵa dd conoimiento dentífica 5a ed. México : Q uinto Sol, 1985. $445 \mathrm{p}$.

Natoli, Joseph P. "Librarianship as a human science: theory, method and application". Library Resegrch no. 4 (1982): 163174.

Neill, Samuel D. Dilemmas in the study of infomation: exploing the boundaries ofinformationsience - New Y ork : G reenwood Press, 1992. xv, 184 p. - (Contributions in librarianship and information science; 70). - ISBN 0-313-27734-6. p. 140-155.

Newton-Smith, W. H. Laracionalidaddela dienaa Barcelona : Paidós, 1987.309 p.

Oldrey, D avid. El arcodi conoimienta introducciónala historia delafiloscfía y mudodogóa dela dena/ / tr. Ferran Vallespinas y Carlos Duarte ; rev. ed. David Casacubierta. - Barcelona : Crítica, 1993. 607 p. - (Serie general ; 228). - ISBN 84-7423-579-0.

Ortiz González, Leoncio. Dicianario de lógica México : IPN, c1995. 400 p.

Pérez Ransanz, Ana Rosa. "Modelos de cambio científico". La aenia: etructura ydesardllo ed. Ulises Moulines. Madrid : Trotta, 1993. 234 p. pp. 181-202.

Powell, Ronald R. Basicrearch mothods for libraians. New Jersey : Ablex, 1985. $188 \mathrm{p}$.

Primo Yúfera, Eduardo. Introduciónalainvestigaaónäentíficayteeno lógica Madrid : Alianza Editorial, 1994. 399 p. (Alianza Universidad). 
Quantitative mothoos in libranianship standards, reserch, managment / ed. Irene Braden Hoadley. Conneticut : G. P., 1974. 270 p.

Ravichandra Rao, I. K. Quantitative mthoods for library and information science New York : John Wiley, 1983. 271 p.

Rendón Rojas, Miguel Ángel. Basesteénicasyfilosóicasdelabibidteedoǵa MéxiCo : UNAM, CUIB, 1997. $132 \mathrm{p}$.

- -. "Metodología de la investigación en bibliotecología". InvestigadónBiblio teedógica: biblidteedogáa, ardhivdoǵa, doumentacón einformaaón v. 10. no. 21 (1996): 27-29.

Rojas L, O ctavio G. "La investigación y el desarrollo integral de la bibliotecología en la región". Memriadd SeninariopreConferenia IFLA sdreecura aón para la investigaión einvestigaión para la eduracoón Bogotá : ALEBCI, 1983. 2 v. : 49-67.

Sander, Susana. "El problema de la investigación bibliotecológica norteamericana : una revisión (1930-1960)”. Invetigacoón Biblidteedógica: ardivonomáa, biblictedogóa, einfomaaión v. 8, no. 16 (1994): 20-24.

Shera, Jesse H. Introductiontolibrarysaience basicdements of librarysaence Colorado : Libraries : Libraries Unlimited, 1976. 208 p.

Torrett, Roberto. "El método axiomático". La denia : etructura y desardlo D e. C. Ulises Moulines. Madrid : Trotta, 1993. 234 p. (Enciclopedia Iberoamericana de Filosofía ; 4): 89-110.

Watofky, Marx W. Introdurciónalafilosofía dela denaia 3a ed. México : Alianza Editorial, 1986. 679 p. (Alianza editorial textos ; 30).

Wold dhanges Thomas Kuln and thenature of sience/ ed. by Paul Horwich. Massachussetts : Bradford Book : The MIT Press, 1993. - vi, 356 p. ISBN 0-262-08216-0.

Wright, H. Curtis. "Inquiry is science and librarianship". Jaumal of Library Histary. no. 13 (1978): 250-264. 